\title{
Differences in Physical Activity Participation Between University Employees With and Without a Worksite Health Promotion Program
}

\author{
Lisa J. Leininger ${ }^{1}$, Debra Harris ${ }^{2}$, Susan Tracz ${ }^{2}$, James E. Marshall ${ }^{2}$ \\ ${ }^{1}$ California State University, Seaside \\ ${ }^{2}$ California State University, Fresno
}

\begin{abstract}
The purpose of this study was to assess differences in physical activity (PA) among university employees with and without a formal health promotion program. Three state university campuses without health promotion programs and four campuses with a program participated in this study. PA participation was assessed via survey to all campus employees. PA was compared for those with $(n=426)$ and without a program $(n=371)$. The results indicated that there was no significant difference $(\mathrm{p}>.05)$ in the amount of vigorous PA days per week between those with $(\mathrm{M}=1.87 \pm 2.29)$ and without a program $(\mathrm{M}=1.6 \pm$ 1.87).There was no significant difference ( $\mathrm{p}>.05$ ) in the amount of moderate PA days per week between those with $(M=2.18 \pm 2.43)$ and without a program $(M=1.88 \pm 2.03)$. There were significant differences $(\mathrm{p}<.05)$ for walking days per week, with the employees with a program having the highest number of days $(\mathrm{M}=4.06 \pm 3.57)$ compared to those without a program $(\mathrm{M}=3.38 \pm 2.28)$. Overall, findings indicate that presence of a health promotion program was only associated with more walking days per week. Therefore, programs must strive to increase moderate intensity PA participation, perhaps through more innovative means, in order to improve the health of their employees.
\end{abstract}

(C) 2013 Californian Journal of Health Promotion. All rights reserved.

Key Words: health promotion programs, physical activity

\section{Introduction}

Worksite health promotion programs have increased in prevalence since the 1980's. With employees spending significant amounts of time at work, the worksite can be an effective place for positive health behavior change. These programs have become prevalent due to the high rates of chronic, hypokinetic diseases that are present in the United States, and a rise in medical costs associated with these diseases. Therefore, the ultimate goal of any worksite health promotion program is to improve the health of employees, increase productivity and help manage rising health care costs (Chenoweth, 2011). Increasing physical activity (PA) levels is imperative to meeting the goals of worksite health promotion programs.

\section{Physical Activity}

PA participation is vital to the prevention and treatment of the many lifestyle related diseases that are top causes of morbidity and disability in the United States, including cardiovascular diseases and cancers. Individuals who participate in regular, moderate intensity PA reduce the risk of developing and dying from these leading causes of illness in the United States (United States Department of Health and Human Services [USDHHS], 2002). PA reduces the risk of dying from heart disease and developing high blood pressure, and helps manage blood glucose levels (Kahn et al., 2002).

The USDHHS document entitled Healthy People 2020 (2010) suggests that PA is a leading health indicator. However despite the known benefits, most Americans do not engage in regular PA. Kahn et al. (2002) report that only $25 \%$ of American adults acquire the USDHHS's 
recommended amount of $\mathrm{PA}$, which at the time of this study was 150 minutes of moderate intensity activity per week (2008). Data from the National Health Interview Survey show that nearly $40 \%$ of adults over 20 years old reported no leisure time PA (CDC, 2010), which is defined as exercise, sports, or hobbies that incorporate PA and movement. Despite the efforts of many health care professionals, PA habits have remained unchanged for the past decade (Haines, Davis, Rancour, Robinson, Neel-Wilson, \& Wagner, 2007).

\section{Worksite Health Promotion}

Worksite health promotion programs have been established nationwide to help increase PA participation, in an effort to increase positive health behaviors, increase morale and productivity of employees and decrease employer health care costs. Companies and institutions, including college campuses nationwide, are implementing health promotion programs for employees (Reger, Williams, Kolar, Smith, \& Douglas, 2002).

According to the Report from the 2000 Joint Committee on Health Education and Promotion Terminology (2001), health promotion is "any planned combination of educational, political, environmental, regulatory or organizational mechanisms that support actions and conditions of living conducive to the health of individuals, groups or communities" (p. 101). By the year 2000 , nearly $90 \%$ of companies with over 50 employees had a health promotion program (Haines et al., 2007).

Benefits to the employee include improved health indicators, an increase in PA participation and increased morale. In fact, worksite health promotion programs are often considered a top benefit in conjunction with insurance and other employee incentives (Chenoweth, 2011). Benefits to the employer are a decrease in health care costs, along with happier, healthier employees.

Several studies indicate that individuals who participate in health promotion programs have improved health related outcomes such as reductions in adiposity, high blood pressure, and high blood glucose and blood lipid levels (Fisher \& Fisher, 1995; Haines et al., 2007; Moxley, 1990; Perryman \& Beerman, 1997; White \& Jacques, 2007). White and Jacques (2007) assessed the effectiveness of a 12-week program on employee risk factors for heart disease, including weight, body composition, blood pressure, cholesterol levels, triglycerides, and blood glucose. Results indicated significant differences for the pre-post intervention measurements for total cholesterol, triglycerides, and weight. In addition, there was a significant correlation between self-reported attendance in program offerings and improvement in cholesterol levels. The researchers concluded that the health promotion program was effective in reducing cardiovascular disease risk (White \& Jacques, 2007).

One of the most important institutional benefits is major cost savings for the employer (Aldana, Merrill, Price, Hardy, \& Hager, 2005). These cost savings include health care costs and abseentism, as well as increases in employee productivity. Heinen and Darling (2009) reviewed Baptist Health South Florida, a large health care system with 12,000 employees that incurs large savings due to their program (Heinen \& Darling, 2009). Their results indicate that substantial health care savings can be realized through comprehensive interventions, including PA interventions. The analysis of their medical claims from the 324 regular participants in their program demonstrated a drop in medical costs, compared to those who did not participate.

\section{Worksite Health Promotion Program on University Campuses}

Studies of worksite health promotion programs specifically at university campuses have been limited. Haines et al. (2007) evaluated the effects of a 12-week walking program conducted at a large mid-western university. Researchers found that improvements in health measures were "noted from beginning to the end of the study" (p. 223). There was a statistically significant overall reduction in BMI, however, remaining measurements were not statistically significant.

Another 12-week pilot study at a Health Sciences University recently evaluated health 
outcome measures of participants (TougerDecker, O'Sullivan- Maillet, Byham-Gray, \& Stoler, 2008). The results indicated statistically significant reductions in weight, BMI, waist circumference, waist to hip ratio, cholesterol and blood pressure, which accounts for nearly every variable studied. The authors concluded that health promotion programs can be a positive addition to the university worksite.

\section{Purpose of the Study}

The purpose of this study was to evaluate differences in PA between university employees with a health promotion program and those without a program. Although research has demonstrated benefits to both the employee and employer, little research has been conducted with university employees and have evaluated their use of worksite health promotion programs. In addition, at the time of publication, the researchers were not aware of any studies that were conducted on many major university campuses in California, where physical inactivity and lifestyle related disease are a major public health issue, comparable to rates across the United States. In addition, college campuses in California employ thousands across the state.

The workplace can be an effective arena for positive health behavior change and universities employ many individuals in the state of California. Therefore, many universities have health promotion offerings for their employees. The current study focused on administration, faculty and staff at universities in California in an effort to evaluate health promotion programs effect on physical activity participation. The researchers predicted that those university employees with a health promotion program would report more vigorous and moderate intensity PA days, as well as more walking days, than those without a program.

\section{Methods}

\section{Sample}

Twenty-three large California university campuses were targeted as the worksites for the study. After contact with all campuses, seven agreed to participate. Three campuses without a formal health promotion program and four campuses with health promotion programs participated in the employee survey.

The four campuses with a health promotion program vary in size. The total number of employees was 754, 1,167, 2,013 and 3,196, (California State University, 2011) with survey return rates of $2.5 \%, 16.7 \%, 9.6 \%$ and $2.7 \%$, respectively. The campuses that participated that do not have a health promotion program also vary in size, with the total number of employees of 1,292, 1,165 and 907 with survey return rates of $2.8 \%, 14.3 \%$ and $21.3 \%$ respectively. All administration, faculty and staff who had an active email account were invited to participate in the study through completion of an electronic survey.

Despite varying in total number of employees, it is important to note that each campus with a health promotion program had elements of all three levels according to O'Donnell's program level classifications. These included education and awareness activities, as well as classes and facility accessibility. Most importantly, each program had a PA component to their program. All four had walking program competitions and offered fitness facilities to their employees.

\section{Data Collection}

Site contacts were established for all campuses. Typically, the site contact was the administrator of the health promotion program, an employee in human resources, or an employee in the online services department. This site administrator assisted the research investigators by sending the employee survey to all employees via electronic communication. All current faculty, staff and administration from each site were sent an email that included a link to the electronic survey. All employees responded on a volunteer basis, were informed of all procedures and consent was obtained prior to participation in the study. The study was approved by the Human Subjects Review Board at each participating university.

\section{Measures}

Inquiries regarding PA were part of a larger survey that also inquired about nutrition habits. 
All PA was self-reported in days per week in which they participated in various activities. The International Physical Activity Questionnaire (IPAQ) was the assessment tool used when constructing the electronic survey. The IPAQ was developed by an international consensus group beginning in 1998, with the purpose of developing a valid and reliable self-reported measurement tool that could be administered in several countries (Craig et al., 2003). Walking days and vigorous and moderate PA days were assessed as separate line items, with the employee choosing how many days they participated in each type of PA. The independent variable in this study was presence of a health promotion program. The dependent variables were number of vigorous intensity PA days, number of moderate intensity PA days and number of walking days per week.

For each category of PA, the employee was instructed to only report PA that was over 10 minutes in duration, and to exclude activities of daily living. The 2008 Physical Activity Guidelines were used to assess intensity of PA. Vigorous activity was described as "higher intensity activities, such as running or cycling at fast pace or lifting very heavy weights. Typically, during high intensity activity a conversation would be difficult to conduct." Moderate PA was described as "lower intensity activity, such as brisk walking or light weight lifting, and in general you could hold a conversation while performing the activity." Employees were also asked to recall the number of days per week in which they walked for more than 10 minute at a time, and to not include walking days in moderate PA day reporting.

\section{Statistical Analysis}

All data analysis was done with Statistical Package for the Social Sciences (SPSS) 20.0. The alpha level for statistical significance was set at $0.05(\alpha=.05)$. To assess differences in PA participation among employees with programs versus those without programs, a multivariate analysis of variance (MANOVA), was calculated. Due to the multiple dependent variables, the MANOVA was utilized to test for differences between groups to reduce Type I error. The independent variable was presence of a health promotion program while the dependent variables were the number vigorous activity days, number of moderate intensity PA days and number of walking days per week. Preliminary analyses, Pearson correlations, indicated a weak relationship between vigorous activity and gender, but no there was no relationship between any other dependent variables and gender, ethnicity or age. Therefore, covariates were not utilized in the analysis.

\section{Results}

A total of 891 employees from all campuses responded to the employee survey. Due to the low response rate, the generalizability of the results may be limited, as many of the demographics reflected in the current study differ from the gender and ethnicity distribution of the campuses included. Of the 891 total respondents, 877 had usable data, with 14 $(1.50 \%)$ missing. The total number of male respondents was $258(29.00 \%)$. The total number of female respondents was 619 $(69.50 \%)$.

Of the $868(97.40 \%)$ who indicated their ethnicity, Blacks accounted for $2.20 \%$ of respondents $(\mathrm{n}=20)$, Asians accounted for $3.40 \%$ of the participants $(n=30)$, Whites accounted for $74.10 \%$ of all respondents $(\mathrm{n}=660)$, Hispanics accounted for $13.70 \%(\mathrm{n}=$ 122), East Indian respondents included $0.80 \%$ of the population $(n=7)$, and people who indicated that their ethnicity was other than those listed accounted for $3.30 \%$ of respondents $(n=29)$. There were no reported Native American respondents.

For the age range 18-29 years, respondents accounted for $10.10 \%(\mathrm{n}=90)$ of the sample size; 30-39 years were $17.28 \%(\mathrm{n}=154)$; ages $40-49$ accounted for $23.12 \%(\mathrm{n}=206)$; ages 50 59 accounted for $29.85 \%(n=266)$; ages $60-69$ accounted for $13.13 \%(\mathrm{n}=117)$; ages $70-79$ accounted for $0.80 \%(\mathrm{n}=8)$; and there was one respondent over the age of 80 , who accounted for $0.10 \%$ of respondents. Those who chose not to respond accounted for $5.50 \%(n=49)$ of the 
participants.

Faculty comprised $35.10 \%$ of the respondents (n $=313)$, staff accounted for $51.70 \%$ of the respondents $(\mathrm{n}=461)$, administration accounted for $10 \%$ of respondents $(n=89)$, and $2.50 \%$ of respondents were classified as other $(\mathrm{n}=22)$. Those who chose not to respond accounted for $0.70 \%(n=6)$ of the participants.

Table 1 shows the mean and standard deviations for each group. The MANOVA testing for differences between those with and without a health promotion program and vigorous $\mathrm{PA}$, moderate PA and walking days per week was significant (Hotelling's Trace $=.015, F(2,793)=$ $4.073, \mathrm{p}=.007)$, indicating that at least one of the dependent variables was significantly different. Table 1 also includes the univariate results for differences in PA days among employees with a program and without a program. Univariate results indicated that there was no significant difference in the amount of vigorous $\mathrm{PA}$ between those with $(\underline{\mathrm{M}}=1.87 \pm$ 2.29) and without a program $(\underline{M}=1.6 \pm 1.87)$. There was no significant difference in the amount of moderate PA between those with $(\underline{M}=$ $2.18 \pm 2.43$ ) and without a program

Table 1

Means, Standard Deviations and Univariate Results of Physical Activity Days for Campuses With and Without Health Promotion Programs

\begin{tabular}{|c|c|c|c|c|c|c|c|c|c|}
\hline \multicolumn{10}{|c|}{ Program Status } \\
\hline & \multicolumn{2}{|c|}{$\begin{array}{c}\text { With } \\
(\mathrm{N}=426)\end{array}$} & \multicolumn{2}{|c|}{$\begin{array}{c}\text { Without } \\
(\mathrm{N}=371)\end{array}$} & \multicolumn{2}{|c|}{$\begin{array}{c}\text { Total } \\
(\mathrm{N}=797)\end{array}$} & \multicolumn{3}{|c|}{ Univariate Results } \\
\hline & $M$ & $S D$ & $M$ & $S D$ & $M$ & $S D$ & $F$ & $p$ & $\mathrm{Eta}^{2}$ \\
\hline $\begin{array}{l}\text { Vigorous } \\
\text { Activity Days }\end{array}$ & 1.87 & 2.29 & 1.60 & 1.87 & 1.74 & 2.11 & 3.23 & .072 & .004 \\
\hline $\begin{array}{l}\text { Moderate } \\
\text { Activity Days }\end{array}$ & 2.18 & 2.43 & 1.88 & 2.03 & 2.04 & 2.25 & 3.36 & .067 & .004 \\
\hline Walking Days & 4.06 & 3.57 & 3.38 & 2.42 & 3.74 & 3.11 & 9.36 & .002 & .012 \\
\hline
\end{tabular}

Note: The multivariate results were significant $\left(T^{2}=.015, F=4.073, p=.007\right)$

$(\underline{\mathrm{M}}=1.88 \pm 2.03), F(1,796)=3.36,, \mathrm{p}=.067$, $\operatorname{Eta}^{2}=.004$.

However, the univariate results for walking days was significant, $F(1,796)=9.36, p=.002, \mathrm{Eta}^{2}$ $=.012$, with the employees with a program having the highest number of days $(\underline{M}=4.06 \pm$ $3.57)$ compared to those without a program $(\underline{\mathrm{M}}=$ $3.38 \pm 2.28)$ although there was a low effect size (.012). Therefore, these results in part support the research hypothesis. The researchers hypothesized that there would be a significant difference in vigorous intensity PA, moderate intensity PA and walking days.

\section{Discussion}

Results indicated that there were no significant differences in vigorous or moderate PA days per week between the employees who had a health promotion program and those without a program. However, there was a significant difference in the number of days that the employees walked for over 10 minutes, with the employees with a health promotion program present reporting more days.

It is important to note that the four universities with health promotion programs included in this study were similar in terms of construction and programming options. For instance, each of the four sites offered a health fair each year, along with several educational opportunities and wellness activities. All four programs emphasized PA as their main program, and each site organized a walking program or competition during the semester of the study. In addition, 
each site had exercise facilities available. The facility offerings did differ in location, cost and accessibility, although all were considered low cost and easily accessible while on campus. In fact, three of the four sites had multiple facilities that employees had access to throughout the day.

There was a significant difference in the amount of walking participation, with the employees at a health promotion program campus reporting more walking days than those without a program. However, it is important to note that the effect size was small and the Eta squared was .012, indicating that only $1.2 \%$ of the variance in walking was accounted for by presence of a health promotion program. Therefore, although statistically significant, these results may lack practical significance. In other words, the greater amount of walking days for those with a program should not be regarded as a profound difference from those without a health promotion program.

These results are important in terms of evaluating the potential impact that health promotion programs may have for the employer and its employees. For instance, many times funding and other support may be dependent on the outcomes of a program. The results of this study indicate that the presence of a health promotion program may not lead to increased PA days. Therefore, the program's impact on health outcomes, specifically PA participation may be limited. This is inconsistent with previous research that indicates increased PA with a health promotion program.

Based on the results of the current study, campuses with health promotion programs should take this information into account to improve their programs and increase PA levels in hopes of increasing health outcomes for their employees and thus capitalizing on benefits of programs for the employer. Program improvement may be accomplished through known best practices, an increase in funding and staff or innovative programming options.

In a recent review of health promotion programs, results indicated that only about half of employers were successful in enticing more employees to participate in programming (Huber \& Pense, 2006). Researchers also found that $51 \%$ of employers also reported drawing the same, small group of employees to each program. This may certainly be a reason that this study did not show greater physical activity for employees with a health promotion program. It may be that those who participate in physical activity do so with or without external encouragement. Therefore, future efforts should focus on recruiting new participants to change health behaviors.

According to Huber and Pense (2006), successful efforts to increase numbers of participants include increasing promotion materials, developing programs based on health risk assessments, internet posting, online program registration, and personal communications to employees. Taitel et al. (2008) suggest that participation in programs may be increased through higher incentives, communication and support from administration. Some more innovative ideas proposed by Taitel et al. include identifying "champions" that are recognized in organization and may be influential to others to participate in programming.

\section{Limitations}

The return rates from each campus were low. Further studies should be conducted to increase return rates and increase sample size at each university site. Alternately, fewer sites could be studied with a more concerted effort at each site to increase return rates.

Another limitation of the study was the structure and implementation of administering an electronic survey on the campuses differed. Each campus has its own policy and procedures for such a case. Many of the campuses initially contacted were unable to participate due to strict policies concerning solicitation of employees or did not accept surveys from off campus researchers.

Overall, those campuses that allowed direct, personal emails to be sent by the health promotion program coordinator had greater return rates than those who have bulletin board 
or online newsletter services. Two of the three campuses without health promotion programs also allowed a listserv email from an administrator, with relatively good return rates.

Another limitation of the study is that the survey is self-report measures for program participation, PA amounts, which historically have moderately accurate ratings. However, survey distribution was the most feasible to include many employees from several campuses in the study.

\section{Implications of the Study}

Ample research indicates the benefits of health promotion programming to both the employee and employer (Aldana et al., 2005; Heinen \& Darling, 2009; Haines et al., 2007; Thompson \& Wolf, 2001). However, much less is known about employee PA habits on varying campuses. Due to the fact that this study indicates that those with a health promotion program do not participate in PA more than those without a program, it is imperative for program administrators to work to improve and continue to evaluate their programs.

With research and implementation of best practices, such as communication, marketing and creating a high level of physical and social support, health promotion employees must continue their work to increase PA levels among their employees. This is imperative to strive towards the goal of improving the health of employees, as PA is beneficial in the prevention and treatment of many lifestyle-related diseases.

Research should continue on health promotion programs at universities to get a better sense of the impact on PA levels and other positive health behaviors. Further studies may want to focus their effort on fewer campuses and evaluate PA participation with activity logs, so the researcher can best determine amount and intensity of exercise. However, researchers may also attempt to increase the sample size from each campus with the survey method, in order to look for stable and meaningful differences.

Overall findings of this study indicate that if health promotion programs are to carry out their mission of improved health behaviors and increased PA levels, best practices must be implemented and further research must be conducted. Helping employees to be physically active will ultimately lead to sustaining long term behavior change of employees in order to see improvement in leading health indicators.

\section{References}

Aldana, S., Merrill, R., Price, K., Hardy, A., \& Hager, R. (2005). Financial impact of a comprehensive multisite workplace health promotion program. Preventive Medicine, 40(2), 131-137.

Calfornia State University (2011). Employee Profiles, Fall 2011. Retrieved from: www.calstate.edu/hr

Centers for Disease Control and Prevention. (2010). National health and nutrition examination survey. Retrieved from http://www.cdc.gov/nchs/nhanes.htm

Chenoweth, D. (2011). Worksite Health Promotion. Champaign, IL: Human Kinetics.

Craig, C., Marshall, A.L., Sjostrom, M., Bauman, A.E., Booth, M.L., Ainswowth, B.E., Pratt, M., Ekelund, U., Yngve, A., Sallis, J.F., \& Oja, P. (2003). International physical activity questionnaire:12-country reliability and validity. Medicine \& Science in Sports \& Exercise, 35(8), 1381-1395.

Fisher, S., \& Fisher, M. (1995). Development, implementation, and evaluation of a health promotion program in a college setting. Journal of American College Health 44(2), 8183. 
Haines, D., Davis, L., Rancour, P., Robinson, M., Neel-Wilson, T., \& Wagner, S. (2007). A pilot intervention to promote walking and wellness and to improve the health of college faculty and staff. Journal of American College Health, 55(4), 219-225.

Heinen, L., \& Darling, H. (2009). Addressing obesity in the workplace: The role of employers. Milbank Quarterly, 87(1), 101-122.

Huber, J., \& Pense, C. (2006). (Eds.). Secrets to wellness and health promotion ROI. Manasquan, NJ: Health Resources Publishing.

Kahn, E., Ramsey, L., Brownson, R., Heath, G., Howze, E., Powell, K.,...Corso, P. (2002). The effectiveness of interventions to increase physical activity. American Journal of Preventive Medicine, 22(4S), 73-107.

Moxley, L. (1990). The development and impact of an experimental student affairs employee wellness program. Research in Higher Education, 31(3), 211-233.

Perryman, S., \& Beerman, K. (1997). Know your numbers: Comparing participants and nonparticipants in a university health screening program. Journal of American College Health, 46(2), 87-91.

Reger, B., Williams, K., Kolar, M., Smith, H., \& Douglas, J. (2002). Implementing universitybased wellness: A participatory planning approach. Health Promotion Practice, 3(4), 507-514.

Report on the 2000 Joint Committee on Health Education and Promotion Terminology. (2001). American Journal of Health Education, 32(2), 14.

Taitel, M.S., Haufle, V., Heck, D., Loeppke, R., \& Fetterrolf, D. (2008). Incentives and other factors associated with employee participation in health risk assessments. Journal of Occupational and Environmental Medicine, 50(8), 863-872).

Thompson, D., \& Wolf, A.M. (2001). The medical-care cost burden of obesity. Obesity Reviews, 2(3), 189-197.

Touger-Decker, R., O'Sullivan-Maillet, J., Byham-Gray, L., \& Stoler, F. (2008). Wellness in the workplace: A 12-week wellness program in an academic health sciences university. Topics in Clinical Nutrition, 23(3), 244-255.

U.S. Department of Health and Human Services. (2010). Healthy People 2020. Rockville, MD.

U.S. Department of Health and Human Services. (2008). 2008 Physical activity guidelines for Americans. Retrieved from http://www.health.gov/paguideline.

U.S. Department of Health and Human Services. (2002). Prevention makes common cents. Retrieved from http://aspe.hhs.gov/health/prevention/

White, K., \& Jacques, P. (2007). Combined diet and exercise intervention in the workplace: Effect on cardiovascular disease risk factors. American Association of Occupational Health Nurses Journal, 55(3), 109-114. 
Author Information

*Lisa J. Leininger, Ed.D.

California State University, Monterey Bay

Department of Kinesiology

100 Campus Center

Seaside, CA 93955

Email: lleininger@csumb.edu

Phone: (831) 582-3962

Debra Harris, Ph.D.

California State University, Fresno

Department of Social Work

Fresno, CA 93740

Susan Tracz, Ph.D.

California State University, Fresno

Educational Research and Administration Department

Fresno, CA 93740

James E. Marshall, Ph.D.

California State University, Fresno

Kremen School of Education and Human Development Fresno, CA 93740

* corresponding author 